\title{
Quantitative Analysis of FeMo Alloys by X-Ray Fluorescence Spectrometry
}

\author{
Sung-Mo Jung \\ Graduate Institute of Ferrous Technology, Pohang University of Science and Technology, Pohang, Korea \\ Email: smjung@postech.ac.kr
}

Received 25 June 2014; revised 2 August 2014; accepted 18 August 2014

Copyright (C) 2014 by author and Scientific Research Publishing Inc. This work is licensed under the Creative Commons Attribution International License (CC BY). http://creativecommons.org/licenses/by/4.0/

c) (i) Open Access

\begin{abstract}
A quantitative analysis method of molybdenum in FeMo alloys by X-ray spectrometry using borate fusion technique was compared with that with pressed pellet. The complete pre-oxidation of FeMo alloys for the preparation of homogeneous fused discs was achieved by employing an automated fusion machine equipped with specially designed $\mathrm{O}_{2}$-blowing nozzles, which used lithium tetraborate as flux with the addition of lithium nitrate $\left(\mathrm{LiNO}_{3}\right)$ as oxidizer. The calibration curves of Mo and Fe were used in the quantitative analysis of standard materials and unknown plant samples with satisfactory accuracy and precision, utilizing the corrections of the matrix effects and line overlap. It was confirmed that the newly proposed method of preparing fused glass discs of FeMo alloys can replace the conventional wet chemical analyses requiring the labor intensive and time consuming procedure.
\end{abstract}

\section{Keywords}

FeMo Alloys, X-Ray Fluorescence Spectrometry, Borate Fusion, Pre-Oxidation, Calibration Standard, Quantification

\section{Introduction}

As it has been required to develop and produce high performance steels for automotive, construction, engineering steel, offshore, packaging and so on, it is necessary to do in-depth research on new steel grades which improve physical properties such as high strength, high toughness, good corrosion resistance, and good formability [1] [2]. Because the physical properties of steel is directly determined by its microstructure, special treatments are applied to the steels for alloy composition adjustment, grain size control, steel cleanliness, deformation/texture control and control of segregation/intermetallic compound. In particular, the addition of ultrapure ferroallloys to liquid steel is of great essence for microalloying to provide specific mechanical properties to steel grade 
[3]. One of the important alloying elements is molybdenum which is charged to liquid steel in the form of FeMo alloys. It is of great importance to accurately and reproducibly analyze Mo in FeMo alloys to control the final concentration of Mo in the ultimate steel grade to be aimed.

Most of the quantifications of Mo in FeMo alloys have been relied on wet chemical analysis such as $\mathrm{KMnO}_{4}$ titration [4]. However, wet chemical analysis is labor-intensive and time-consuming work, and the use of toxic chemicals is very hazardous to humans. It has been demanded to convert the wet chemical analysis into the routine and automated instrumental analysis employing X-ray fluorescence (XRF) spectrometry by developing the borate fusion technique. In the case of fused disc preparation of ferroalloys, most of the ferroalloys mixed with oxidizer are stored in a muffle furnace of about $800^{\circ} \mathrm{C}$ overnight for preliminary oxidation of the samples, which also takes great care and time [5] [6]. Therefore, a rapid and automated fusion equipment is being attempted to be used for the integration of the complicated and stepwise procedure into one simple and routine preparation of fused discs for ferroalloys.

The current investigation aims to compare the XRF analysis by borate fusion method with that by pressed pellet from viewpoints of the sensitivity, accuracy and reproducibility for the analysis of Mo in FeMo alloys. Accordingly, it will be confirmed whether the conventional wet chemical analysis of molybdenum in FeMo alloys can efficiently be replaced with the XRF analysis using the homogeneous fused glass discs prepared in an automated fusion system equipped with specially designed $\mathrm{O}_{2}$-supplying apparatus.

\section{Experimental}

\subsection{X-Ray Fluorescence Spectrometer}

Bruker-AXS S4 Pioneer wavelength dispersive X-ray fluorescence spectrometer (WD-XRF) was employed in this research. The XRF was equipped with an end window Rh-target X-ray tube of the maximum output of $4 \mathrm{~kW}$ and eight diffracting crystals of various d-spacings. The measuring conditions and goniometer settings were programmed by the Bruker-AXS software "Spectra Plus" and summarized in Table 1. This application software was employed to calculate theoretical "variable alpha coefficients". Alpha coefficients correct the absorption or enhancement of secondary radiation due to inter-element or matrix effects. The XRF was employed to measure the calibration standards in scan mode, and the optimal positions of highest signal intensities of peak and background were determined by overlapping the data files measured in scan mode. Subsequent XRF calibration and measurement were carried out in fixed mode.

\begin{tabular}{|c|c|c|c|c|}
\hline Spectral lines & \multicolumn{2}{|c|}{ Mo } & \multicolumn{2}{|c|}{$\mathrm{Fe}$} \\
\hline Sample preparation & Pressed pellet & Borate fusion & Pressed pellet & Borate fusion \\
\hline Spectral line & \multicolumn{2}{|c|}{ Mo LA1-HR-Min (High resolution line) } & \multicolumn{2}{|c|}{ Fe KA1-Maj (Majority line) } \\
\hline Mode & Atmospheric He & Vacuum & Atmospheric He & Vacuum \\
\hline Tube voltage $(\mathrm{kV})$ & 27 & 30 & 50 & 50 \\
\hline Tube current (mA) & 112 & 77 & 10 & 8 \\
\hline Crystal (2d, Å) & \multicolumn{2}{|c|}{ PET (8.752) } & \multicolumn{2}{|c|}{$\operatorname{LiF} 200(4.026)$} \\
\hline Collimator aperture (degree) & \multicolumn{2}{|c|}{0.23} & \multicolumn{2}{|c|}{0.23} \\
\hline Detector & \multicolumn{2}{|c|}{ Flow } & \multicolumn{2}{|c|}{ Scintillation } \\
\hline Adjusted peak (2 $\theta)$ & 76.360 & 76.327 & 57.527 & 57.537 \\
\hline Wavelength $(\AA)$ & \multicolumn{2}{|c|}{5.405} & \multicolumn{2}{|c|}{1.936} \\
\hline Position of background ( $2 \theta)$ & 77.612 & 74.542 & 58.470 & 58.346 \\
\hline
\end{tabular}




\subsection{Availability of Calibration Standards of FeMo Alloys for XRF Analysis}

$\mathrm{X}$-ray fluorescence spectrometry is a comparative measurement, which means that the quantification of elements is based on predetermined calibration curves constructed by using calibration standards. Therefore, the analysis of unknown FeMo alloys strongly depends on the availability of certified reference materials. In this study, nine calibration standards were used and their certified concentrations are listed in Table 2. The three Fe compositions marked by asterisks were analyzed in lab by ICP-AES (inductively coupled plasma-atomic emission spectroscopy) since the certified Fe concentrations were not available. This procedure is essential for XRF calibration because it is necessary to establish XRF calibration curves covering wide range of compositions and to simultaneously meet the requirement of variable alpha coefficient method for matrix corrections where the sum of all the compositions should lie in between 95 and 105 mass\% for the calibration standards used [7].

\subsection{XRD Patterns of a FeMo Alloy and a Calibration Standard}

In most cases, X-ray fluorescence spectrometry analyzes the concentrations of cations or anions (e.g. Fe, Mo, etc.) and then converts them to those of compounds which are assumed to be most likely to exist in a sample. In order to identify the existing phases of Mo in FeMo alloys, X-ray diffraction analyses were performed for a FeMo alloy and a calibration (HC18605) as shown in Figure 1. The primary phase of Mo in both samples was estimated to be $\mathrm{Fe}_{3} \mathrm{Mo}$. This indicates that the effect of Mo existing in different forms on the matrix correction in XRF analysis will be minimized in case the pelletized FeMo alloys and calibration standards are directly measured by XRF. Therefore, it is straightforward to deal with Mo content in FeMo alloys and calibration standards where Mo only exists in the form of $\mathrm{Fe}_{3} \mathrm{Mo}$ in the pressed pellet.

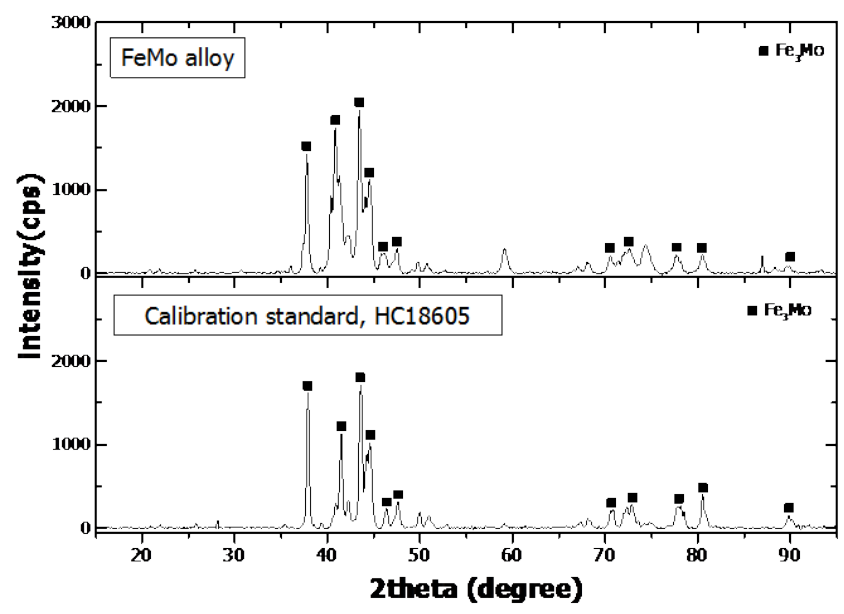

Figure 1. X-ray diffraction pattern of a FeMo alloy used in secondary steelmaking process and one of the standard reference materials employed in the current study.

Table 2. Compositions of calibration standards of FeMo alloys available in markets.

\begin{tabular}{|c|c|c|c|c|c|c|c|c|c|c|}
\hline \multirow{2}{*}{$\begin{array}{l}\text { Calibration } \\
\text { standards }\end{array}$} & \multicolumn{10}{|c|}{ Composition (mass \%) } \\
\hline & Mo & Fe & C & $\mathrm{Si}$ & $\mathrm{S}$ & $\mathbf{P}$ & Mn & $\mathbf{N i}$ & $\mathrm{Cr}$ & $\mathbf{C u}$ \\
\hline HC18605 & 55.78 & 42.53 & 0.015 & 0.055 & 0.079 & 0.154 & - & - & - & 0.63 \\
\hline SL20-13 & 60.03 & 38.42 & 0.029 & 0.28 & 0.081 & 0.039 & 0.012 & 0.017 & 0.05 & 0.4 \\
\hline SL20-20 & 62.20 & 36.71 & 0.028 & 0.21 & 0.079 & 0.034 & 0.013 & 0.019 & 0.057 & 0.376 \\
\hline HC26610 & 66.52 & 31.59 & 0.049 & 1.20 & 0.064 & 0.034 & - & - & - & 0.52 \\
\hline SL20-12 & 69.99 & 27.82 & 0.011 & 0.79 & 0.084 & 0.028 & 0.042 & - & - & 0.39 \\
\hline E578-1 & 72.23 & 26.46 & 0.016 & 0.208 & 0.065 & 0.024 & - & - & - & 0.136 \\
\hline SL20-08 & 72.82 & 25.51 & 0.018 & 0.71 & 0.059 & 0.017 & 0.088 & 0.02 & 0.039 & 0.378 \\
\hline SL20-06 & 73.83 & 24.03 & 0.031 & 0.252 & - & 0.086 & 0.07 & 0.905 & 0.018 & 0.134 \\
\hline SL20-10 & 78.09 & 20.66 & 0.026 & 0.161 & 0.092 & 0.017 & 0.010 & 0.112 & 0.035 & 0.464 \\
\hline
\end{tabular}




\section{Results and Discussion}

\subsection{Preparation Procedure of Pressed Pellet of FeMo Alloys}

Powdered samples constitute one of the most frequently used specimen forms in x-ray spectrometry. Powders are more often analyzed as a pressed pellet. To achieve a uniform small particle size, the pulverization is carried out. It is not unreasonable to expect a relationship between fluorescent $\mathrm{x}$-ray intensity and particle size since $\mathrm{x}$-rays can only penetrate and emerge from a finite depth beneath the surface. Although there have been several theoretical effects of particle size and size distribution upon x-ray spectrochemical analysis results [8] [9], the qualitative effects of particle size on the spectral line intensity can be alleviated in most cases by reduction of the entire sample to sub-10 $\mu \mathrm{m}$ particles. The detailed procedure is as follows:

- The nine calibration standards have been pulverized for about $1 \mathrm{~h}$ by employing a planetary ball mill technique so that the particle size was decreased to approximately less than $10 \mu \mathrm{m}$.

- For the XRF measurement of samples in the form of pressed pellets, boric acid powder was first lightly packed to a $40 \mathrm{~mm}$ aluminum cup inside a $42 \mathrm{~mm}$ ID cylindrical die.

- An accurately weighed portion of each sample was added in sufficient amount to cover the top of the boric acid powder.

- A plunger was inserted into the die and hydraulic pressure was applied in a briquetting press (Herzog, maximum pressure 40 ton).

- Only one face of sample material in the resulting sample disc encased in an aluminum dish was exposed to $\mathrm{X}$-ray radiation for analysis.

- Briquettes formed in this manner remained intact after repeated analyses, provided they were stored in a dessicator.

\subsection{Calibration for Pelletized FeMo Alloys and Evaluation of Their Validity}

The calibration curves of Mo and Fe in the form of pressed pellet are presented in Figure 2 based on the calibration conditions summarized in Table 3. Each calibration curve was obtained by applying matrix corrections with the help of variable alpha coefficient method and line overlap compensation. The linearity of Mo and Fe calibration curves were improved by removing three and two points out of the entire calibration standards, respectively. From the viewpoints of standard deviation resulted from the calibration curves in Table 3 and squared correlation coefficient values in Figure 2, the case of Fe shows better results in comparison with that of Mo. It is believed that this is ascribed to the higher sensitivity of Fe-KA1-Major line compared with Mo-LA1-HR (high resolution)-Major line, which will be discussed later. From the relative linear correlations between corrected $\mathrm{X}$-ray intensity and concentrations of Mo and Fe for the investigated compositional range of each component, it was believed that XRF calibration using pressed pellet can approximately be applied for the chemical analysis of FeMo alloys.

Table 3. Calibration data for Mo and Fe in the original sample.

\begin{tabular}{|c|c|c|c|c|}
\hline Spectral line & \multicolumn{2}{|c|}{ Mo LA1-HR-Maj } & \multicolumn{2}{|c|}{ Fe-KA1-Maj } \\
\hline Sample preparation & Pressed pellet & Borate fusion & Pressed pellet & Borate fusion \\
\hline Absorption correction & \multicolumn{2}{|c|}{ Variable alpha } & \multicolumn{2}{|c|}{ Variable alpha } \\
\hline Intensity model & \multicolumn{2}{|c|}{ Net intensity } & \multicolumn{2}{|c|}{ Net intensity } \\
\hline Minimization target (mass \%) & $\begin{array}{c}\text { Absolute error, } \\
6 \text { standards } \\
\text { from } 55.78 \text { to } 73.83\end{array}$ & $\begin{array}{l}\text { Absolute error, } \\
9 \text { standards from } \\
55.78 \text { to } 77.64\end{array}$ & $\begin{array}{l}\text { Absolute error, } \\
7 \text { standards from } \\
20.66 \text { to } 42.53\end{array}$ & $\begin{array}{l}\text { Absolute error, } \\
9 \text { standards from } \\
20.66 \text { to } 42.53\end{array}$ \\
\hline Standard deviation (mass \%) & 1.53 & 0.45 & 0.557 & 0.400 \\
\hline Sensitivity (KCps/\%) & 1.668 & 0.212 & 10.16 & 0.367 \\
\hline Corrected intensity offset & $-87.8 \mathrm{KCps}$ & - & $-18.79 \mathrm{KCps}$ & 0.017 KCps \\
\hline Line overlap correction & None & S KA1 & Mn KB1 & - \\
\hline
\end{tabular}



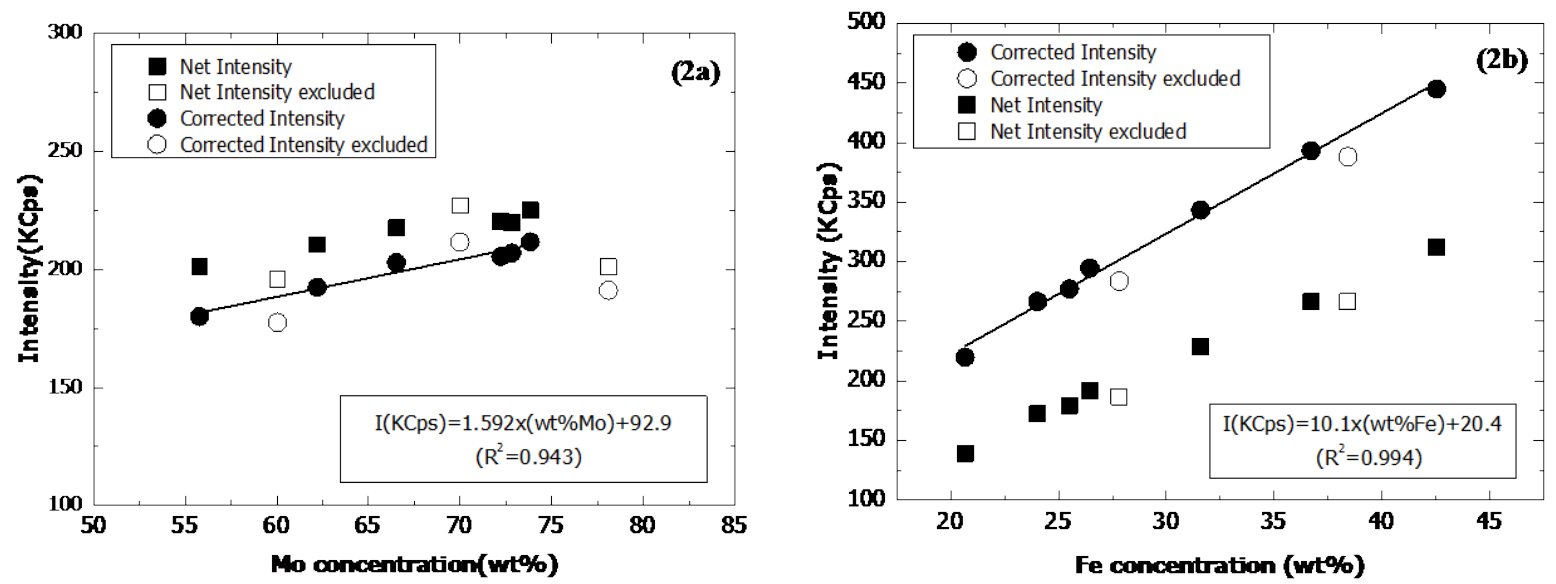

Figure 2. XRFCalibration curves of Mo (a) and Fe (b) using pressed pellet with matrix effects and line overlap corrections.

In order to evaluate the validity of the XRF calibration curves, three repeated XRF measurements were made on one of the calibration standards used. As shown in Table 4, XRF analyses of the calibration standard (SL20-20) are quite precise since all the precision RSD (RSD $(\%)=($ standard deviation)/average $\times 100)$ values for Mo and Fe values from each constituent show less than about $0.5 \%$. Therefore, the XRF calibration curves using the calibration standards can be utilized for the quantitative chemical analysis of Mo and Fe in FeMo alloys with acceptable precision for the quality control of FeMo production process.

\subsection{Preparation Procedure of Glass Discs by Borate Fusion Technique}

The borate fusion technique has been employed to eliminate particle size and mineralogical effects. In the case of ferro alloys, due to the interaction between the sample and Pt crucible during fusion, the pre-oxidation of the sample in a muffle furnace for overnight requires a time-consuming and labor-intensive work as previously mentioned. Therefore, the specially designed $\mathrm{O}_{2}$-supplying nozzles were additionally installed over the platinum crucibles in order to ensure the complete oxidation of the FeMo samples during the preparation of the fused discs. The detailed preparation procedure of the newly proposed fusion is as follows:

About $10 \mathrm{~g}$ of lithium tetraborate $\left(\mathrm{Li}_{2} \mathrm{~B}_{4} \mathrm{O}_{7}\right)$ were first weighed in a platinum crucible $(5 \% \mathrm{Au} / \mathrm{Pt})$ and then a spatula was used to form a solid wall of the flux.

- $0.11 \mathrm{~g}$ of non-wetting agent $(\mathrm{LiBr})$ was put onto the flux and then $1.5 \mathrm{~g}$ of oxidizer $\left(\mathrm{LiNO}_{3}\right)$ was added into the platinum crucible.

- $0.3 \mathrm{~g}$ of FeMo sample was put in the crucible (dilution factor $=1 / 33$ ) and mixed with the oxidizer. The overall composition and cooling conditions must be such that the end product after cooling is a homogenous glass disc.

- As shown in Figure 3, the platinum crucible containing the flux, non-wetting agent, oxidizer and sample started to be heated up to $615^{\circ} \mathrm{C}$ for $10 \mathrm{~min}$ and then the temperature was slowly raised up to $850^{\circ} \mathrm{C}$ in $\mathrm{O}_{2}$-blowing atmosphere $(1 \mathrm{l} / \mathrm{min})$ for $45 \mathrm{~min}$ for ensuring the complete pre-oxidation of the sample.

- Then the crucible was quickly heated up to $1000^{\circ} \mathrm{C}$ and held at the temperature for $3 \mathrm{~min}$, then being followed by the pouring of the melt into a platinum mold.

- Right after the fused melt started to be naturally cooled down, the rapid cooling was employed by fan cooling from the bottom of the mold for easy release of the quenched disc from the platinum mold.

Infrared spectroscopy has been extensively employed over the years to investigate the glass structures. In particular, borate glasses have been the subject of numerous infrared studies, due to their structural peculiarities [10] [11]. Although sufficient homogenization in liquid borate melt is carried out during fusion, some crystallization might be resulted in the glass disc during cooling. Therefore, the current study employed FT-IR spectroscopy to confirm whether the fused glass is really homogeneous across the disc surface to be exposed to the XRF radiation as shown in Figure 4. That is, the measured data for five positions on the disc by FT-IR spectroscopy indicate that some functional groups (O-B-O ring bending, symmetric and asymmetric stretching) were clearly identified over the specific spectral range, which are typical spectral peaks for borate glasses. As is clearly noticed, 


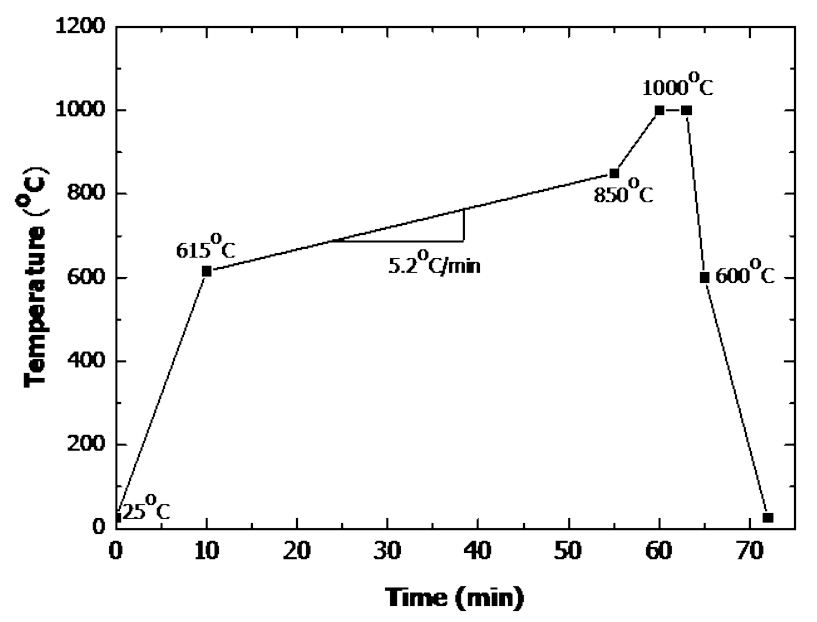

Figure 3. Heating and cooling profiles of the automated fusion system for preparing the fused discs of FeMo alloys using Kanax $\mathrm{K}_{2}$ equipped with $\mathrm{O}_{2}$-supplying nozzles over fusion stations.

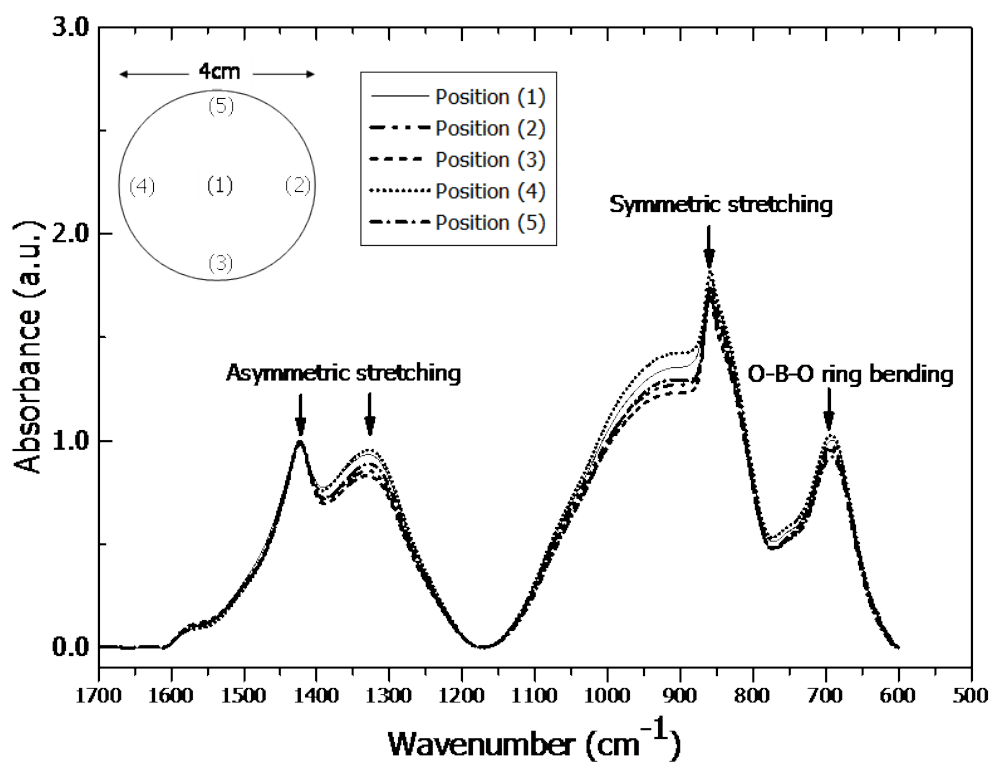

Figure 4. Homogeneity testing of the borate fusion disc of a calibration standard (SL20-20) employing FT-IR spectroscopy.

Table 4. Precision and accuracy tests for a calibration standard (SL20-20) by three XRF measurements using pressed pellet and borate fusion (units: mass\%).

\begin{tabular}{ccccc}
\hline \multirow{2}{*}{ Elements } & \multicolumn{2}{c}{ Mo (Certified conc. $=\mathbf{6 2 . 2 0}$ mass \%) } & \multicolumn{2}{c}{ Fe (Certified conc. = 36.71 mass \%) } \\
\cline { 2 - 5 } & Pressed pellet & Borate fusion & Pressed pellet & Borate fusion \\
\hline Test_01 & 62.87 & 62.07 & 36.73 & 36.41 \\
Test_02 & 62.85 & 62.02 & 36.78 & 36.50 \\
Test_03 & 63.20 & 62.02 & 36.76 & 36.48 \\
Average & 62.97 & 62.04 & 36.76 & 36.46 \\
Standard deviation & 0.19 & 0.02 & 0.03 & 0.04 \\
Precision RSD (\%) & 0.31 & 0.04 & 0.07 & 0.11 \\
\hline
\end{tabular}


there is no remarkable difference among the IR spectra for the five positions. This strongly indicates that the fused disc is homogeneous enough to be used for XRF calibration and analyses and that the newly proposed fusion method of FeMo alloys can be applied for the reliable preparation of borate glass for XRF analysis of FeMo alloys.

\subsection{Calibration for Fused Glass Discs of FeMo Alloys and Evaluation of Their Validity}

The nine calibration standards were fused into glass discs by lithium borate fusion technique, and the calibration curves of Mo and Fe were constructed as shown in Figure 5. Each calibration curve was obtained by applying matrix corrections with the help of variable alpha coefficient method and line overlap corrections. Both of them indicate good linear correlations between corrected X-ray intensity and concentrations of Mo and Fe for the investigated compositional range of each component. Therefore, it is believed that the nine standard materials in the form of fused glass disc can appropriately be applied to XRF calibration for the chemical analysis of FeMo alloys.

As is similar to the case of pressed pellets, the validity of the XRF calibration curves was evaluated by making three repeated XRF measurements on one of the standards (SL20-20) used for XRF calibration. As shown in Table 4, XRF analyses of the FeMo alloy components are quite precise since both the relative standard deviation (RSD) values for Mo and Fe show less than about $0.5 \%$. In Table 4, the analysis results by XRF are comparable to the certified concentrations of Mo and Fe, and can be acceptable considering that small portion of the Mo samples might vaporize during borate fusion process. Therefore, the XRF calibration curves using all the nine standards can be utilized for the quantitative chemical analysis of Mo and Fe in FeMo alloys with acceptable precision and accuracy for the sake of quality control of Mo microalloying in secondary steelmaking processes.

\subsection{Comparison of Sensitivity, Accuracy and Precision between Pressed Pellet and Fused Disc}

In case the borate fusion technique is employed for the sample preparation of X-ray spectrometry, the spectral signal of low concentration of fluorine in stainless steelmaking slags might not be detected due to its low sensitivity [12]. Sensitivity is defined as the fractional change in instrument response per unit change in concentration of the measured element [7]. Accordingly, the slopes of the calibration lines in Figure 2 and Figure 5 correspond to the sensitivities of the selected spectral lines of respective element in FeMo alloys.

In order to figure out how the sample preparation methods affect the sensitivity of each spectral line, the comparison was made between the calibration lines constructed by borate fusion technique and those by pressed pellet. As obviously noticed in Figure 6, the sensitivities of spectral lines of Mo and Fe decreased by about 6 and 27 times, respectively, although the dilution ratio of sample to borate fusion flux is 1 to 33. That is, the sensitivity of Mo LA1-HR line decreased less compared with the dilution ratio and the linearity of the Mo calibra-
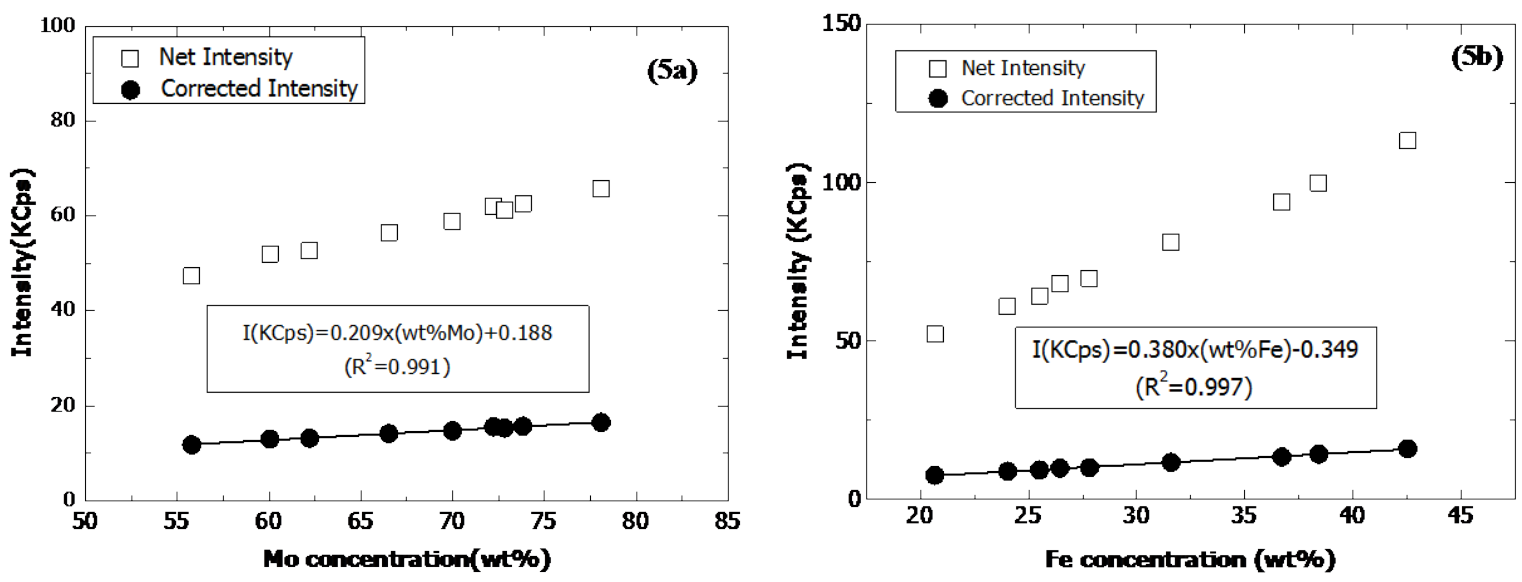

Figure 5. XRFCalibration curves of Mo (a) and Fe (b) using borate fusion technique with matrix effects and line overlap corrections. 
tion line using borate fusion technique was improved as clearly compared in Figure 2 and Figure 5. This indicates that the measured intensity of Mo in the form of borate fused discs was nicely corrected by applying variable alpha coefficients and line overlap calculation against Mo concentration in the calibration standards.

Once the calibration curves of the specific elements in a material are successfully established using the pressed pellet and borate fusion technique, the subsequent step is to actually analyze unknown samples by XRF spectrometry and to compare them with the results certified by wet chemical analysis. As shown in Table 5, two unknown FeMo alloys to be used in secondary steelmaking plants were analyzed by XRF using both the preparation methods and compared with the results obtained by wet chemical analysis of Mo [4]. In both the samples, the analyzed Mo content by XRF using the borate fusion method is much closer to that determined by wet chemical analysis compared with that using pressed pellet. However, from a viewpoint of precision of the data, there is no great difference in the standard deviation between pressed pellet and borate fusion method. Therefore, in the actual operational situation of sophisticated charging of FeMo alloys in secondary steelmaking plants, the suggested preparation using borate fusion for the Mo analysis by XRF could be more reliable in case the rapid and routine analysis of Mo should be carried out. That is, the calibration curves of Mo and Fe can be utilized for the quantitative chemical analyses of Mo and Fe in FeMo alloys depending on the precision and accuracy to be aimed for the quality control of secondary steelmaking process.

\section{Conclusion}

X-ray fluorescence spectrometry of FeMo alloys employing an automated fusion system equipped with $\mathrm{O}_{2}$-supplying nozzles was compared with that using pressed pellet. From the findings, the following conclusions were obtained. From the viewpoints of standard deviations and squared correlation coefficient values resulted from the calibration curves of Mo and Fe using pressed pellet, the case of Fe shows better results in comparison with that of Mo, which is ascribed to the higher sensitivity of Fe-KA1 line compared with Mo-LA1-HR (high resolution) line. The complete oxidation of FeMo alloys by adding oxidant and by employing $\mathrm{O}_{2}$-supplying apparatus made contribution to the preparation of the fused discs, whose homogeneity was confirmed by FT-IR spectroscopy. The measured intensity of Mo in the form of borate fused discs was nicely corrected by applying variable alpha coefficients and line overlap calculation against Mo concentration in the calibration standards.

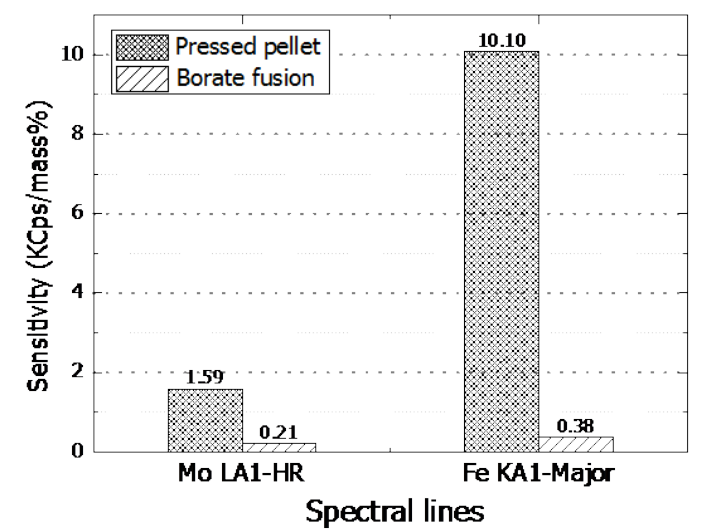

Figure 6. Comparison of the sensitivities of each spectral line (Mo and $\mathrm{Fe}$ ) affected by sample preparation methods (Pressed pellet vs Borate fusion).

Table 5. Comparison of the analysis results of plant unknown FeMo alloys by wet chemical analysis and XRF measurement.

\begin{tabular}{cccc}
\hline \multirow{2}{*}{ Unknown samples } & $\begin{array}{c}\text { Mo conc. by wet chemical } \\
\text { analysis (mass \%) }[4]\end{array}$ & \multicolumn{2}{c}{ Mo content by XRF (mass \%) } \\
\cline { 2 - 4 } & 60.99 & $60.42\left( \pm 0.05^{*}\right)$ & $60.90\left( \pm 0.13^{*}\right)$ \\
FeMo1 & 64.33 & $62.24\left( \pm 0.14^{*}\right)$ & $63.99\left( \pm 0.11^{*}\right)$ \\
\hline
\end{tabular}

* represents the standard deviations produced by three repeated measurements using the respective preparation methods. 


\section{Acknowledgements}

Financial support from POSCO is gratefully appreciated. And the author is grateful to Mr. Wan-Ju Kim and Gyu-Hae Hwang for their assistance in the sample preparations and chemical analyses in this work.

\section{References}

[1] Bhadeshia, H.K.D.H. (2010) Nanostructured Bainite. Proceedings of the Royal Society A, 466, 3-18. http://dx.doi.org/10.1098/rspa.2009.0407

[2] Fan, D.W., Kim, H.S. and De Cooman, B.C. (2009) A Review of the Physical Metallurgy Related to the Hot Press Forming of Advanced High Strength Steel. Steel Research International, 80, 241-248.

[3] ASM International Handbook Committee (1990) Metals Handbook. Vol. 1, 10th Edition, ASM International, Materials Park, 126-137,140-159.

[4] JIS (1982) Methods for Chemical Analysis of Ferromolybdenum (JISG 1317). Tokyo, 704-705.

[5] Automated Fusion Technology Inc. (2001) A Handbook of Fusion Methods. Bayswater, 1-12.

[6] Buhrke, V.E., Jenkins, R. and Smith, D.K. (1998) A Practical Guide for the Preparation of Specimens for X-Ray Fluorescence and X-Ray Diffraction Analysis. John Wiley \& Sons, New York, 41-44.

[7] Jenkins, R., Gould, R.W. and Gedcke, D. (1995) Quantitative X-Ray Spectrometry. Marcel Dekker, New York, 372387.

[8] Claisse, F. and Samson, C. (1962) Heterogeneity Effects in X-Ray Analysis. Springer, 335-354.

[9] Hunter, C.B. and Rhodes, J.R. (1972) Particle Size Effects in X-Ray Emission Analysis: Formulae for Continuous Size Distributions. X-Ray Spec, 1, 107-111.

[10] Kamitsos, E.I., Patsis, A.P., Karakassides, M.A. and Chryssikos, G.D. (1990) Infrared Reflectance Spectra of Lithium Borate Glasses. Journal of Non-Crystalline Solids, 126, 52-67. http://dx.doi.org/10.1016/0022-3093(90)91023-K

[11] Verhoef, A.H. and den Hartog, H.W. (1995) Infrared Spectroscopy of Network and Cation Dynamics in Binary and Mixed Alkali Borate Glasses. Journal of Non-Crystalline Solids, 182, 221-234. http://dx.doi.org/10.1016/0022-3093(94)00555-9

[12] Jung, S.-M., Sohn, I. and Min, D.-J. (2010) Chemical Analysis of Argon-Oxygen Decarburization Slags in Stainless Steelmaking Process by X-Ray Fluorescence Spectrometry. X-Ray Spec, 39, 311-317. 
Scientific Research Publishing (SCIRP) is one of the largest Open Access journal publishers. It is currently publishing more than 200 open access, online, peer-reviewed journals covering a wide range of academic disciplines. SCIRP serves the worldwide academic communities and contributes to the progress and application of science with its publication.

Other selected journals from SCIRP are listed as below. Submit your manuscript to us via either submit@scirp.org or Online Submission Portal.
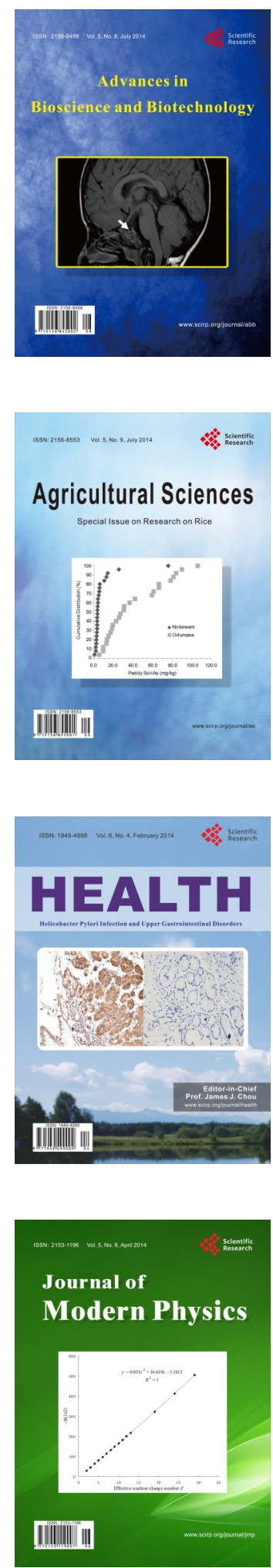
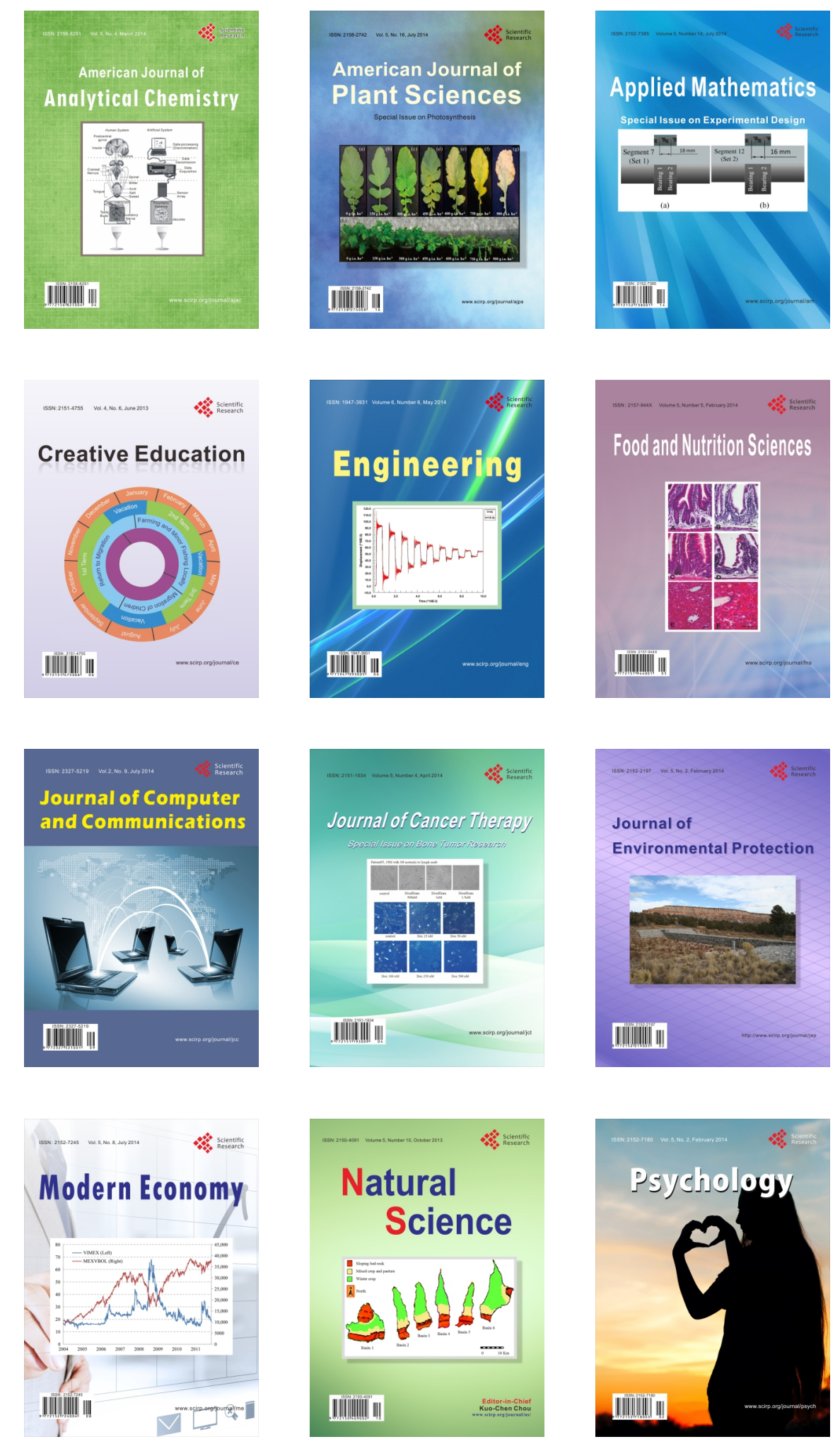